\title{
Copper Deficiency Myeloneuropathy Mimicking Subacute Combined Degeneration Following Bariatric Surgery
}

\author{
Ismail $\mathrm{II}^{{ }^{*}}$, Yassin $\mathrm{OM}^{2}$ and Foad $\mathrm{SS}^{3}$
}

${ }^{1}$ Neurology Specialist, Department of Neurology, Alexandria University

${ }^{2}$ Associate Professor, Department of Neurology, Alexandria University

${ }^{3}$ Associate Lecturer, Department of Neurology, Alexandria University

*Corresponding author: Ismail II, Neurology Specialist, Department of Neurology, Alexandria University, E-mail: dr.ismail.ibrahim2012@gmail.com

Citation: Ismail II, Yassin OM, Foad SS (2014) Copper deficiency myeloneuropathy mimicking subacute combined degeneration following bariatric surgery. J Neurol Neurol Disord 1(1): 104 . doi: 10.15744/24544981.1.104

Received Date: November 14, 2014 Accepted Date: December 23, 2014 Published Date: December 30, 2014

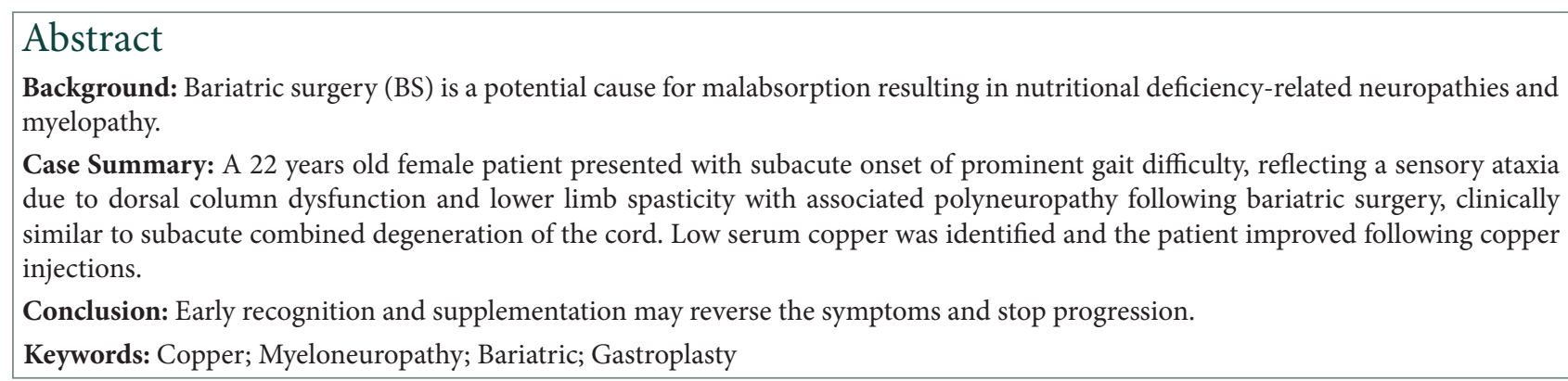

\section{Introduction}

Copper deficiency is a recent but increasingly recognized cause of neurological impairment. Gastric surgery for weight reduction is a potential cause for malabsorption or limited absorption resulting in nutritional deficiency-related neuropathies and myelopathy including copper deficiency myeloneuropathy [1]. We present a 22 years old female with copper deficiency myeloneuropathy post Bariatric surgery.

\section{Case Report}

A 22 years-old female patient presented to our hospital with subacute onset of gait disturbance, weakness and parasthesia of both lower limbs that progressed to parasthesia of both upper limbs within one week with no motor involvement of the upper limbs.

The patient's condition started $1 \& 1 / 2$ month following Vertical Banded Gastroplasty (VGB) for weight reduction. Patient had recurrent attacks of severe vomiting and constipation post-operatively before the onset of neurological manifestations. There was no associated cognitive, ocular, bulbar, cranial nerve or sphincter affection. The onset was not associated with fever or trauma and past medical history of the patient was irrelevant with no history of similar condition. Patient was overweight with Body Mass Index (BMI) of $\left(38.1 \mathrm{~kg} / \mathrm{m}^{2}\right)$.

Neurological examination revealed a fully conscious and oriented patient with moderately ataxic-spastic gait. Motor assessment showed weakness of both lower limbs of MRC grade 3 with a pyramidal distribution. There was no upper limb weakness. Deep Tendon Reflexes (DTRs) examination revealed increased knee jerks and absent ankle jerks in the lower limbs. In the upper limbs biceps, triceps and brachioradialis tendon jerks were normal. Tone was normal in the upper limbs and moderately increased in both lower limbs. The deep sensory examination revealed impaired perception of proprioception and vibration in the distal lower limbs in both peripheral nerve and posterior column manner and to a mild degree in the upper limbs. Superficial sensory examination showed reduced perceptions of pinprick and touch in a glove \& stocking distribution. No sensory level was present over the trunk. Romberg's Sign was positive and Plantar Reflex was extensor bilaterally.

Laboratory studies showed mild microcytic hypochromic anemia; Hb: 11.4 (Ref 12-16.5 g/dL), PVC: 0.31 (Ref 0.36-0.47), MCH: 80.1 (Ref 82.0-97.0 fL), MCH: 24.6 (Ref 27.0-33.0 pg), MCHC: 309 (Ref 32.0-36.0 g/dL). Platelet and WBC count were within normal limits. Blood film was normal. ESR, Liver Functions, Renal Functions, Electrolytes, Thyroid Function, vasculitic work up and Protein Electrophoresis were normal. Serum B12 and Homocystine levels were also normal. Methylmalonic acid (MMA) detection was not available.

Magnetic resonance imaging (MRI) of the spine (cervical, dorsal and lumbosacral) plus MR imaging of the brain were normal. 
Electrophysiological study showed sensory-motor axonal polyneuropathy associated with generalized radiculopathy affecting both lower limbs, much more sensory than motor. Somatosensory evoked potential (SSEP) of lower limbs showed delayed response. Both upper limbs were mildly affected and there was no evidence of polymyositis, myopathy or Motor Neuron Disease (MND).

Our patient was diagnosed initially as "Subacute Combined Degeneration (SCD) of the Cord" and received intramuscular hydroxycobalam injections ( $1000 \mathrm{mcg}$ ) daily for 2 weeks with no improvement.

Serum Copper deficiency was identified after 2 month from the onset of her symptoms and it was found low: $37.72 \mu \mathrm{g} / \mathrm{dL}$ (normal 76-152 $\mu \mathrm{g} / \mathrm{dL}$ ), Serum Ceruloplasmin was also low: $5.6 \mathrm{mg} / \mathrm{dL}$ (normal 22.9 to $43.1 \mathrm{mg} / \mathrm{dL}$ )

The diagnosis of "Copper Deficiency Myeloneuropathy" was made supported by the history of Bariatric Surgery, low serum copper and ceruloplasmin levels and the electrophysiological findings.

Our patient received parenteral Copper Supplementation with marked improvement both subjectively and objectively within 2 weeks of therapy. Serum Copper and Ceruloplasmin has returned back to normal levels one month after supplementation.

\section{Discussion}

Our patient presented with a clinical and electrophysiological picture similar to SCD that was not responsive to vitamin B12 supplementation. Low serum copper was identified and the patient showed improvement following copper injections. Our findings were consistent with other case reports [2] and case series where copper deficiency caused such clinical presentation in absence of radiological and hematological findings [1,3].

Gastric surgery for weight loss (Bariatric Surgery) is an increasingly pursued mean of weight control. It is a potential cause for malabsorption or limited absorption surface resulting in nutritional deficiency-related neuropathies and myelopathy. Nutritional complications following BS may occur for a variety of reasons including reduced dietary intake, reduced gastric acid, inadequate intrinsic factor secretion, or lack of nutrient exposure to the duodenum [4]. Neurological complications following BS in two prior large studies are reported to occur in 5\% -16\% of patients according to a review of 18 surgical series reported between 1976 - 2004 [5]. Myelopathy was the most frequent and disabling problem followed by peripheral neuropathy. Encephalopathy and polyradiculoneuropathy were acute and early complications [6].

Neurological complications following BS have been linked to the several specific deficiencies including Vitamin B1 (thiamine), Vitamin B6 (pyridoxine), Vitamin A, Vitamin B12 and Copper [7].

Copper is an essential trace element, and diseases in animals and humans associated with altered copper levels have been recognized since the early 20th century. An ataxic disorder caused by nutritional copper deficiency termed (swayback) was first described in sheep in 1937 and has since been found in other domestic and wild animals [8].

In humans, nutritional deficiency of copper is rare. The well recognized disorders of hereditary copper deficiency are "Menkes disease" and "Occipital horn syndrome" where there is failure to mobilize copper absorbed into the mucosal cells due to mutations in the ATP7A gene, which encodes copper-transporting ATPase [9]. Acquired copper deficiency could result from different factors including BS, Zinc Toxicity (Zinc induces the synthesis of metallothionein in the enterocytes), Dietary insufficiency, Iron supplementation, Celiac Disease and Total parental nutrition [10].

The hematologic manifestations of copper deficiency are well described, but it was not until recently in 2001 that copper deficiency was associated with neurological manifestations. The first report of copper deficiency myelopathy was in a 46-years-old woman with previous gastrectomy [2].

A notable range of times between the gastric procedure and onset of neurological symptoms has been reported to range from few months to 24 years [3].

Diagnosis depends on detecting low serum copper, ceruloplasmin (with or without hematological findings) and 24 hours urinary copper with addition to imaging and electrophysiological studies [3]. Spinal MRI can show T2-signal hyperintensity preferentially in cervical dorsal column usually involving more than 2 vertebral bodies [11]. Brain MRI may show hyperintense lesions similar to those seen in SCD. Both spinal and brain MRI could be normal in such cases [12], which was similar to our case.

Electrodiagnostic testing is helpful in the diagnosis of patients with copper deficiency myeloneuropathy. Median and tibial SEPs typically show slowing in central somatosensory pathways, although proximal peripheral slowing can be seen with median nerve stimulation. NCS and EMG abnormalities can range from normal to moderately abnormal with the overall pattern of abnormalities suggesting a mild distal axonal sensorimotor peripheral neuropathy. This peripheral neuropathy does not appear to be severe enough to explain the sensory ataxia. In contrast, the results of SEP studies suggest that either proximal peripheral or central impairment in sensory conduction is the basis for the sensory ataxia. The evidence of impaired central sensory conduction correlates with MRI findings of abnormal signal change that might be seen in the dorsal column. It is particularly important to consider SEP testing in patients with significant sensory loss, who have normal or only minimally abnormal sensory NCS findings [13]. In our case NCS and SSEP were helpful to confirm our diagnosis. 


\section{Conclusion}

Copper deficiency post BS could be presented with myeloneuropathy without hematological manifestations. Despite recognition of copper deficiency as a possible cause of neurological symptoms following BS it's usually overlooked. Early diagnosis and treatment of copper deficiency may reverse the symptoms and stop progression.

\section{References}

1. Kumar N, Crum B, Petersen RC, Vernino SA, Ahlskog JE (2004) Copper deficiency myelopathy. Arch Neurol 61: 762-66.

2. Schleper B, Stuerenburg HJ (2001) Copper deficiency-associated myelopathy in a 46-year-old woman. J Neurol 248: 705-6.

3. Kumar N, Gross JB, Ahlskog JE (2004) Copper deficiency myelopathy produces a clinical picture like subacute combined degeneration. Neurology 63: 33-9.

4. Fragoso YD, Brooks JB, Gomes S, Lin K, Lopes J et al. (2012) Neurological complications following bariatric surgery. Arq Neuropsiquiatr, 70 : $700-3$.

5. Koffman BM, Greenfield LJ, Ali II, Pirzada NA (2006) Neurologic complications after surgery for obesity. Muscle Nerve 33: 166-76.

6. Juhasz-Pocsine K, Rudnicki SA, Archer RL, Harik SI (2007) Neurologic complications of gastric bypass surgery for morbid obesity. Neurology 68: 1843-50.

7. Juhasz-Pocsine K, Rudnicki SA, Archer RL, Harik SI (2007) Neurologic complications of gastric bypass surgery for morbid obesity. Neurology 68: 1843-50.

8. Bennetts HW, Chapman FE (1937) Copper deficiency in sheep in WesternAustralia: A preliminary account of the aetiology of enzootic ataxia of lambs and anaemia of ewes. Aust Vet J 13: 138-49.

9. Kaler SG, Gallo LK, Proud VK, Percy Ak, Mark Y, et al. (1994) Occipital horn syndrome and a mild Menkes phenotype associated with splice site mutations at the MNK locus. Nat Genet 8: 195-202.

10. Jaiser SR, Winston GP (2010) Copper deficiency myelopathy. J Neurol 257: 869-81.

11. Kumar N, Ahlskog JE, Port JD, Klein CJ (2006) Imaging features of copper deficiency myelopathy: a study of 25 cases. Neurorad 48 : 78 -83.

12. Goodman BP, Chongb BW, Patel AC, Smith BE (2006) Copper Deficiency Myeloneuropathy Resembling B ${ }_{12}$ Deficiency: Partial Resolution of MR Imaging Findings with Copper Supplementation. AJNR 27: 2112-4.

13. Goodman BP, Bosch EP, Ross MA, Smith BE, Dodicket DD et al. (2009) Clinical and electrodiagnostic findings in copper deficiency myeloneuropathy. J Neurol Neurosurg Psychiatry 80: 524-7. 